\title{
A Compact Active Stereovision System with Dynamic Reconfiguration for Endoscopy or Colonoscopy Applications
}

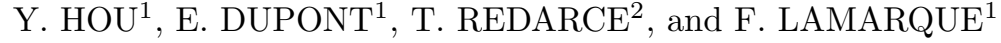 \\ 1 Université de Technologie de Compiègne (UTC), Laboratory Roberval(UMR 7337), \\ 1, rue du Dr Schweitzer 60200 Compiègne, France. yingfan.hou@utc.fr \\ 2 Institut National des Sciences Appliquées de Lyon (INSA-Lyon), Laboratoire \\ Ampère(UMR 5005), 20 av Albert Einstein 69100 Villeurbanne, France. \\ tanneguy.redarce@insa-lyon.fr
}

\begin{abstract}
A new concept of endoscopic device based on a compact optical probe which can capture 3D shape of objects using an active stereovision method is presented. The distinctive feature of this probe is its capability to dynamically switch between two distinct points of view. If the first measurement angle of view does not give results with sufficient quality, the system can switch to a second mode which sets distinct angle of view within less than 25 milliseconds. This feature consequently allows selecting the angle that provides the more useful 3D information and enhances the quality of the captured result.

The instrumental setup of this measurement system and the reconstruction algorithms are presented in this paper. Then, the advantages of this new endoscopic probe are explained with an experimental 3D reconstruction of a coin's surface. Finally, first measurements on a phantom colon are provided. In future works, further miniaturization of the device and its integration into a real colonoscope will be implemented.
\end{abstract}

\section{Introduction}

Recent advances in the technology of medical devices have allowed for the surgeons to provide more precise and reliable diagnosis and for the patient a quicker recovery time and shorter hospital stays. Miniaturization is one of these advances with technologies such as swallowable wireless capsule endoscopy in [1], or more generally the various technologies employed in Minimally Invasive Surgery (MIS) [2]. Many researchers have presented great perspectives of endoscopy or colonoscopy in detecting small objects and minimizing invasiveness at the same time.

Using computer vision algorithm with enhanced endoscopy or colonoscopy devices offers a convenient way to obtain accurate 3D surface information. In [3], Schmalz et al. have proposed an endoscopic 3D scanner with single-shot-colorring pattern structured light, which offers an axial resolution of less than $200 \mu m$ for the reconstructed surface. Maurice et al. [4] have presented a laparoscope 
with real-time organs' surface reconstruction in MIS. These devices are both based on projection of one static pattern.

There is a great variety of methods to achieve $3 \mathrm{D}$ reconstruction in a medical environment, such as fringe projection [5], stereo matching [6], structure from shading [7], shape from motion [8], time of flight [9] etc. Although all these methods have their own pros and cons, it is still a challenging problem to obtain accurate $3 \mathrm{D}$ results in gastrointestinal condition or in MIS.

Active stereovision is one of these methods. Its principle is to project patterns on the measured surface from a first angle of view and to capture the image of the patterns from a second angle of view. Through this triangulation, the 3D depth information of the object is obtained. A review of the active methods for stereovision is proposed in [5]. These active methods can been applied in endoscopic medical devices $[10,11]$.

Although active stereovision methods can extract surface information with good quality, some factors such as surface occlusions, shadows, specular areas or absorptive materials can lead to loss of 3D information during the reconstruction process. One basic principle to overcome some of these difficulties is to capture more images of the object in different positions [12], with the drawback of time consumption.

Another possibility, proposed in this paper, is to dynamically generate two distinct measurement results by switching between projection and capture channels. Combining these two results can be an additional way to enrich the surface information obtained from the measurement.

In order to design a compact system, flexible image guides have been integrated in a compact optical probe. This probe is an improved model of the one presented in [13] and its size is fitted for colonoscopy. The view angle switch is achieved with two miniature digital electromagnetic actuators on witch mobile mirrors are mounted. This type of actuator is chosen because it provides a nonconsuming holding force, which is obtained with magnetic interactions between permanent magnets, at its discrete states without the need of external electrical energy [14]. Another property of the proposed device is its ability to dynamically modify the projected patterns during the measurement to make them suitable for various reconstruction algorithms.

The structure of our system and its measurement principle are described in section 2. Section 3 presents the new designed compact optical probe. Some details on the algorithms are provided in section 4. Measurement results are shown in section 5 and the conclusion and perspectives of our work are finally discussed in section 6 .

\section{System Structure Description}

In standard active stereovision systems, optical and mechanical configurations are generally static. In this section a dynamic system configuration obtained with the integration of miniature digital actuators is presented. This new configuration enhances the 3D reconstruction capability of the proposed system. 
A schematic description of the proposed system is shown in two distinct configurations in Fig.1. As shown in Fig.1(a), a white light source illuminate the DMD (Digital Micro-mirror Device, a projecting device fabricated by Texas Instrument Inc). The patterns generated by DMD go through the lenses and image guide 1 (these image guides are fiber bundle composed of 60000 optical fibers for a total diameter of $1.4 \mathrm{~mm}$, FIGH-60-1200N, fabricated by Fujikura Inc) to enter the compact optical probe. These patterns are then projected onto the objects' surface and reflected back into the compact optical probe. Due to the stereoscopic angle between the two channels of the probe, the projected patterns are deformed and captured by the image guide 2 . These deformed patterns are finally projected on a CCD sensor and depth analysis is performed on the captured images.

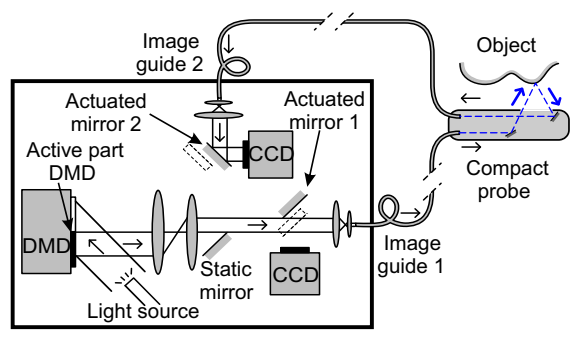

(a)

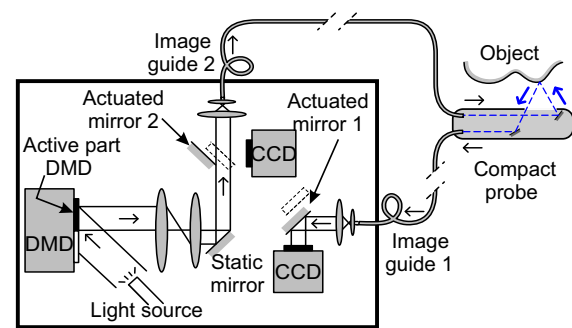

(b)

Fig. 1. Principle of the measurement modes.(a): active mode 1.(b): active mode 2.

In Fig.1(a), only half of the pattern generated by the DMD go through the system by image guide 1 because the other part of the DMD is used on the other mode (Fig.1(b)) to inject the image guide 2. Actuated mirror 1 and 2 are mounted on two digital electromagnetic miniature actuators(each of them have two discrete states) to switch between positive and negative positions. The first mode (Fig.1(a)) is obtained by setting actuated mirror 1 in negative position and by setting the actuated mirror 2 in positive position to reflect the patterns from image guide 2 to the CCD sensor.

The other active stereovision mode is obtained by switching the position of both actuated mirrors (Fig.1(b)). The other half of DMD is used to inject the patterns into image guide 2 after being reflected by static mirror 1 . Another CCD sensor captures the patterns from image guide 1 . In the following sections, the configuration of Fig.1(a) will be called "mode 1" and Fig.1(b) "mode 2".

Even though the object and the measure area are identical in mode 1 and mode 2, the captured images and the reconstruction results are different because the projection and view angles are exchanged between these two modes. It means that some sides of the measured object are more precisely captured with the first mode (i.e. the first view angle of mode 1) and other sides of the object are better measured with the second mode. In the final reconstruction the more 
precise measurements from each mode can be selected to optimize the quality of the result.

\section{Structure of Compact Optical Probe}

To fulfill requirements in gastrointestinal diagnosis, a new compact optical probe was designed. The structure of the probe is shown in Fig.2.

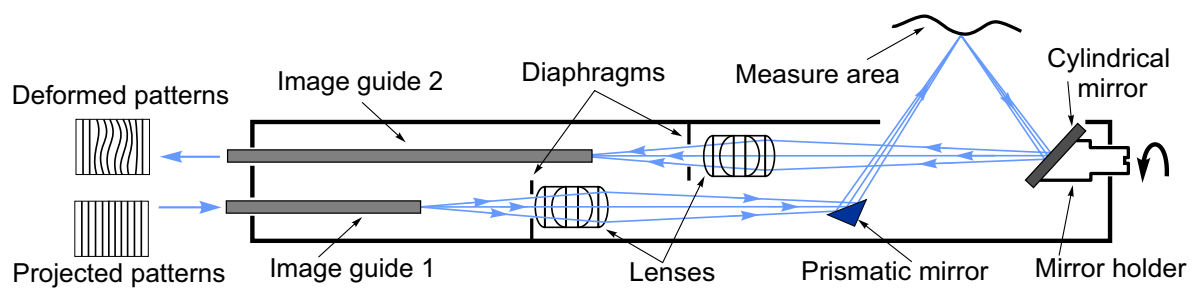

(a)

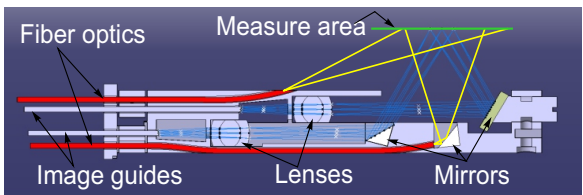

(b)

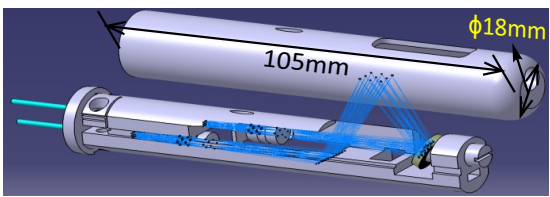

(c)

Fig. 2. Principle structure of compact optical probe.(a): Functional schematic for mode 1.(b): Schematic with two integrated fibers optics and two image guides. (c): Overall view of compact optical probe.

Two image guides are connected with two Steinheil Triplet Achromat Lenses of $6.5 \mathrm{~mm}$ diameter and two diaphragms. Some mirrors are also used in the probe to reflect the patterns either from the image guides to the object or from the object to the image guides. As shown in Fig.2, the measurement area is at the side of the cylindrical probe. This is suitable for measurement in gastrointestinal diagnosis. In such a way, a $360^{\circ}$ esophagus or intestine stripe can be measured by just rotating the probe during the operation. In this compact optical probe, two optical fibers can be added, as shown in Fig.2(c). Non-structured light can be projected directly on the surface of the objects by these optical fibers if the object must be seen without pattern projection. The circular mirror at the end of the probe can be rotated. This allows tuning the reflecting angle to make sure the two channels are capturing or projecting on the same area of the object. The probe size is about $\phi 18 \times 105 \mathrm{~mm}$ and the capture area is about $16 \mathrm{~mm}^{2}$. In future works, this compact optical probe will be enhanced to capture a much larger area, which can be a necessity when the capture of large areas is needed. And the miniaturization will be tried for the size of this probe. 


\section{Reconstruction Algorithm}

Structured light projection techniques are generally classified in three principles [5], [15]: the first one is the spatial neighborhood principle, where generally a unique pattern is projected onto the measure area. This method can be used in dynamic scenes but has difficulties to provide accurate results in presence of shadows or occlusions. The second principle is the direct codification strategy. Each pixel of the projected pattern has a specific color or grey level which is used to detect the corresponding projected pixel. This method is very sensitive to the scene noise and generally request to capture more than one image, which is not suitable in dynamic environments. The third principle is the time multiplexing principle which gives the highest resolution results. A set of patterns are successively projected onto the measure area and the codeword for one pixel is given by the intensity variation between the sequences of values received by the pixel. The resolution of the measurement will increase with the number of projected patterns. The drawback of this method is that it is not applicable to dynamic scenes because of the big number of patterns required.

For our system, the time multiplexing methods is selected to optimize the resolution. In future works, we will determine what quality can be achieved with the projection of a unique pattern.

In the scope of the time multiplexing methods, phase-shift and gray code methods are two possibilities. Our study in [13] showed that the best reconstruction results were obtained with phase shift algorithms, however these algorithms need phase unwrapping and high number of patterns was needed to optimize the axial resolution. Gray code is well suited for lower resolution results, but with fewer projected patterns.

In the following experimental section, phase-shift method is used for accurate measurement on a 10 cents euro coin because more objects' detail information is included in coin's surface, and Gray-code method is applied to do faster measurement on a colon phantom because only surface form information want to be detected.

\section{$5 \quad$ Measurement Results}

Some experimentations were carried out with the presented prototype and the two modes were tested. Firstly, a 10 cent euro coin has been measured to validate the properties of our proposed system (noted Test 1). Then measurements have been applied on a colon phantom to test the device in a medical environment (noted Test 2).

In test 1 , the letter "N" of the "CENT" word has been measured on the euro coin, the results are presented in Fig.3. Phase-shift method was used to obtain a more precise reconstructed 3D model. Vertical line patterns were projected onto the object. In Fig.3(b) and (c), which are the two captured results of the two modes, the vertical line patterns are deformed because of the height of letter "N". Moreover, the orientation of patterns' deformation is opposite between these 


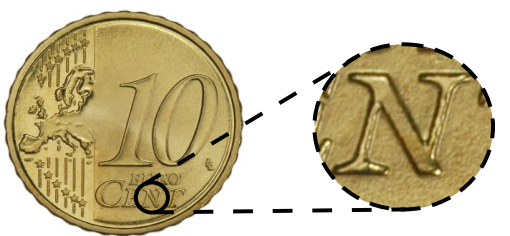

(a)

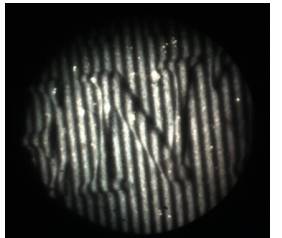

(b)

(c)

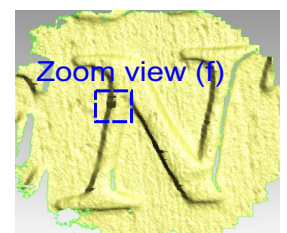

(d)

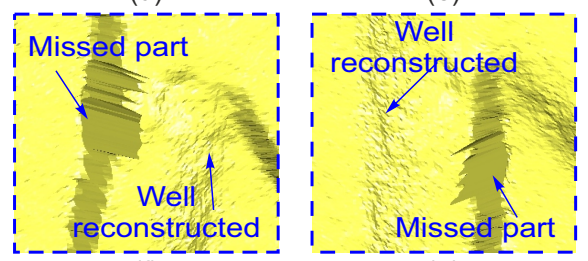

(f)

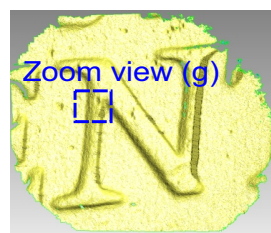

(e)

(g)

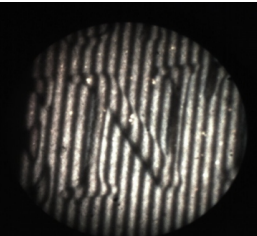

Fig. 3. Experimental measurement results for test 1.(a): measured letter $\mathrm{N}$ from a 10 cent of euro coin. (b) and (c): result of active mode 1 and 2. (d) and (e): reconstruction results of mode 1 and mode 2 with phase shift method. (f) and (g): zoom view of (d) and (e).

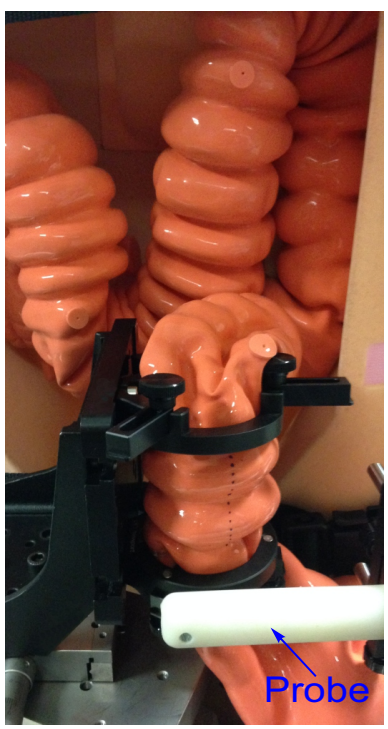

(a)

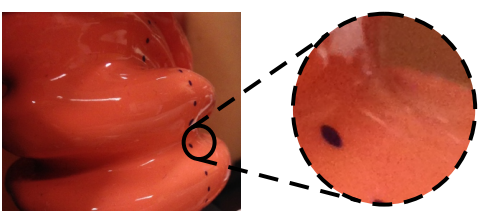

(b)

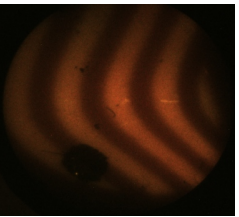

(d)

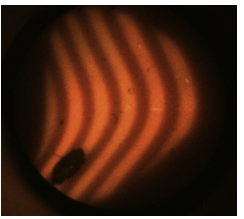

(g)

$(\mathrm{e})$

(h)

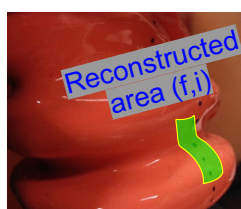

(c)
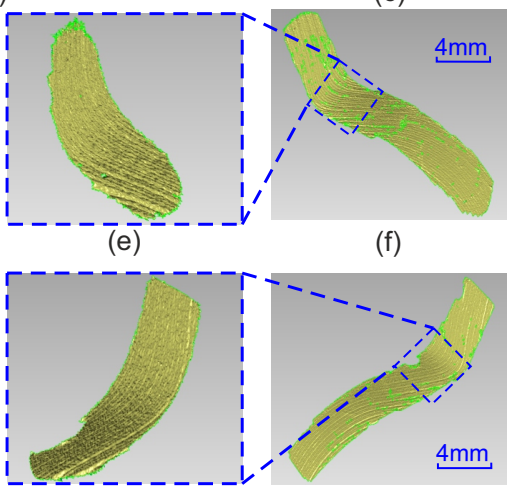

(i)

Fig. 4. Experimental measurement results for test 2.(a): setup for the test 2(the reference points are drawn onto the dummy intestine's surface to make sure series measurement position are in line). (b): external form of dummy intestine is measured in one measurement. (c): 15 measurements are taken to reconstruct a large zone of external form. (d) and (g): result of active mode 1 and 2. (e) and (h): reconstruction results of mode 1 and mode 2 with gray code method. (f) and (i): enlarged measurement area of mode 1 and mode 2 . 
two results: on to the left in Fig.3(b) and on to the right in Fig.3(c). This is the consequence of the two different angle of views in the two active stereovision modes. The final reconstructed 3D models are presented in Fig.3(d) and (e). The left side of letter "N" in Fig.3(d) was not well reconstructed (e.g. the missed part indicated in Fig.3(f)), but this side is well reconstructed in Fig.3(e)(e.g. the well reconstructed part indicated in Fig.3(g)) and vice versa. Using the dual mode reconstruction principle, the final $3 \mathrm{D}$ result can be enriched by combining the 3D models obtained from the two modes.

In test 2 , a colon phantom has been used to measure its shape. For the sake of simplicity, the external form of intestine is measured instead of inside form in this test, by considering the same material they have possessed. The experimental results are shown in Fig.4. Comparing to 32 patterns used for a precise phase-shift measurement in test 1 , gray code method was chosen in this test 2 to do a faster reconstruction with fewer projected patterns (only 8). The reconstructed surface (Fig.4(e) and (h)) shows a small part of the colon captured in these two measurement modes. The measurement area can be enlarged by moving the probe with small increments. 15 more positions were measured with the two modes, and the surface results are stitched in Fig.4(f) and Fig.4(i). The Geomagic Studio software was used to merge the captured point cloud data from the 2 modes and to generate the final reconstructed surface. In a real case, the view angle that provide the best quality of result could be automatically selected to help the surgeons during its surgical operation.

\section{Conclusions}

In this paper, a new miniaturized 3D measuring system able to be reconfigured dynamically between two active stereovision modes is presented. The projection and acquisition channels can be switched to obtain complementary 3D measurements of the same surface. A new compact optical probe was designed to achieve miniaturization. Experimental results on a 10 cent euro coin and on a phantom colon have been presented to demonstrate its feasibility in medical environment. The dynamic system reconfiguration allows to select the best angle of view for the capture and consequently improve the quality of the result.

Due to the fact the prototype is in its preliminary design and experimentation phase, some improvements are planned to enhance its capabilities. The first one is to further miniaturize the device and study its integration into a real colonoscope to realize the measurements on a medical environment. We will also modify the optical setup of the probe to measure larger areas. Other reconstruction methods will be studied to meet the real-time reconstruction condition with a unique pattern projection and consequently no time multiplexing.

\section{References}

1. Cavallotti, C., Piccigallo, M., Susilo, E., Valdastri, P., Menciassi, A., Dario, P.: An integrated vision system with autofocus for wireless capsular endoscopy. Sensors and Actuators A: Physical 156(1) (2009) 72-78 
2. Valdastri, P., Simi, M., Webster III, R.J.: Advanced technologies for gastrointestinal endoscopy. Annual review of biomedical engineering 14 (2012) 397-429

3. Schmalz, C., Forster, F., Schick, A., Angelopoulou, E.: An endoscopic 3d scanner based on structured light. Medical image analysis 16(5) (2012) 1063-1072

4. Maurice, X., Albitar, C., Doignon, C., de Mathelin, M.: A structured light-based laparoscope with real-time organs' surface reconstruction for minimally invasive surgery. In: Engineering in Medicine and Biology Society (EMBC), 2012 Annual International Conference of the IEEE, IEEE (2012) 5769-5772

5. Gorthi, S.S., Rastogi, P.: Fringe projection techniques: whither we are? Optics and lasers in engineering 48(2) (2010) 133-140

6. Cui, H., Dai, N., Liao, W., Cheng, X.: Intraoral 3d optical measurement system for tooth restoration. Optik-International Journal for Light and Electron Optics 124(12) (2013) 1142-1147

7. Wu, C., Narasimhan, S.G., Jaramaz, B.: A multi-image shape-from-shading framework for near-lighting perspective endoscopes. International Journal of Computer Vision 86(2-3) (2010) 211-228

8. Grasa, O.G., Civera, J., Guemes, A., Munoz, V., Montiel, J.: Ekf monocular slam $3 \mathrm{~d}$ modeling, measuring and augmented reality from endoscope image sequences. In: Medical Image Computing and Computer-Assisted Intervention (MICCAI). Volume 2. (2009)

9. Penne, J., Höller, K., Stürmer, M., Schrauder, T., Schneider, A., Engelbrecht, R., Feußner, H., Schmauss, B., Hornegger, J.: Time-of-flight 3-d endoscopy. In: Medical Image Computing and Computer-Assisted Intervention-MICCAI 2009. Springer (2009) 467-474

10. Wong, C., Chen, N., Sheppard, C.: Study on potential of structured illumination microscopy utilizing digital micromirror device for endoscopy purpose. In: Biophotonics, Nanophotonics and Metamaterials, 2006. Metamaterials 2006. International Symposium on, IEEE (2006) 218-221

11. Dupont, E., Lamarque, F., Prelle, C., Redarce, T., et al.: Tri-dimensional optical inspection based on flexible image guide: first step toward 3d industrial endoscopy. In: Proc. of the 11th Biennial Conference on Engineering Systems Design and Analysis. Volume 19. (2012)

12. Cui, H., Liao, W., Dai, N., Cheng, X.: Registration and integration algorithm in structured light three-dimensional scanning based on scale-invariant feature matching of multi-source images. Chinese Optics Letters 10(9) (2012) 091001-091001

13. Dupont, E., Hou, Y., Lamarque, F., Redarce, T.: Binary pattern codification strategies in an active stereoscopic system based on flexible image guides. In: SPIE MOEMS-MEMS, International Society for Optics and Photonics (2013) 86180H$86180 \mathrm{H}$

14. Miao, X., Dai, X., Wang, P., Huang, Y., Ding, G., Zhao, X.: Electromagnetic bistable microactuator fabricated on a single wafer. Micro \& Nano Letters, IET 7(2) (2012) 99-100

15. Salvi, J., Pages, J., Batlle, J.: Pattern codification strategies in structured light systems. Pattern Recognition 37(4) (2004) 827-849 